\title{
Mutation analysis of the Smad3 gene in human osteoarthritis
}

\author{
Jun-Yan Yao ${ }^{1,2}$, Yan Wang ${ }^{3}$, Jing An ${ }^{2}$, Chun-Ming Mao ${ }^{1}$, Ning Hou ${ }^{1}$, Ya-Xin $\mathrm{Lv}^{1}$, \\ You-Liang Wang ${ }^{1}$, Fang Cui ${ }^{1}$, Min Huang ${ }^{3}$, Xiao Yang ${ }^{\star, 1}$
}

${ }^{1}$ Genetic Laboratory of Development and Diseases, Institute of Biotechnology, Beijing 100071, People's Republic of China; ${ }^{2}$ First Military Medical University, Guangzhou 510515, People's Republic of China; ${ }^{3}$ PLA General Hospital, Beijing 100850, People's Republic of China

Osteoarthritis (OA) is the most common joint disease worldwide. Recent studies have shown that targeted disruption of Smad3 in mouse results in OA. To reveal the possible association between the Smad3 gene mutation and human $\mathrm{OA}$, we employed polymerase chain reaction-single strand conformation polymorphism and sequencing to screen mutations in all nine exons of the Smad3 gene in 32 patients with knee OA and 50 patients with only bone fracture. A missense mutation of the Smad 3 gene was found in one patient. The single base mutation located in the linker region of the SMAD3 protein was $A \rightarrow T$ change in the position 2 of codon 197 and resulted in an asparagine to isoleucine amino-acid substitution. The expressions of matrix metalloproteinase 2 (MMP-2) and MMP-9 in sera of the patient carrying the mutation were higher than other OA patients and controls. This is the first report showing that the Smad3 gene mutations could be associated with the pathogenesis of human OA. European Journal of Human Genetics (2003) 11, 714-717. doi:10.1038/sj.ejhg.5201034

Keywords: osteoarthritis; Smad3 gene; mutation; PCR-SSCP

\section{Introduction}

Osteoarthritis (OA) is the most common degenerative arthritis, a type of arthritis that is caused by breakdown of articular cartilage with eventual loss of the cartilage of the joints. ${ }^{1}$ The prevalence of $\mathrm{OA}$ is high and expected to increase in the coming years. ${ }^{2}$ Results of epidemiologic researches indicate that the incidence of symptomatic OA is about $8-9 \%$ in China. ${ }^{3,4} \mathrm{OA}$ is a multifactorial disorder in which aging, genetic, hormonal and mechanical factors are major contributors to its onset and progression, but the molecular mechanism underlying the cartilage degeneration is still largely unknown.

Many studies have shown that transforming growth factor- $\beta$ (TGF- $\beta$ ) signals function as key regulators in bone

*Correspondence: Dr X Yang, Genetic Laboratory of Development and Diseases, Institute of Biotechnology, 20 Dongdajie, Fengtai, Beijing 100071, People's Republic of China. Tel/fax: +86-10-63895937,

E-mail: yangx@nic.bmi.ac.cn

Received 12 November 2002; revised 7 March 2003; accepted 11 April 2003 formation, remodeling and maintenance. The SMAD proteins are downstream mediators of the TGF- $\beta$ signals. ${ }^{5}$ Recently, targeted disruption of Smad3 have revealed that the Smad3 is required for maintaining articular cartilage in the quiescent state by repressing chondrocyte hypertrophic differentiation and regulating matrix molecule synthesis. $^{6,7}$ To check if the Smad3 gene mutations are associated with the pathogenesis of OA in humans, we had analyzed 32 patients with knee osteoarthritis. Mutations in the nine exons of the Smad3 gene were screened using polymerase chain reaction-single strand conformation polymorphism (PCR-SSCP) and consequent DNA sequencing. The activities of matrix metalloproteinase 2 (MMP-2) and MMP-9 in sera of OA patients and controls were also examined by gelatin zymography.

\section{Materials and methods}

Patients

A total of 32 sporadic cases of knee OA and 50 patients with only bone fracture (controls) were studied. The patients 
with knee OA were diagnosed based on international criterion and the controls were excluded by the same criterion. Genomic DNA was extracted from peripheral blood samples using Ready $\mathrm{PCR}^{\mathrm{TM}}$ Blood Genomic Purification System kit (Sino-American Biotechnology Co., China).

\section{PCR-SSCP}

PCR was performed according to the published primers and conditions. ${ }^{8}$ Briefly, gels were routinely run at $5 \mathrm{~V} / \mathrm{cm}$ for $5-6 \mathrm{~h}$ at $4{ }^{\circ} \mathrm{C}$ in $1 \times$ TBE buffer. The gels were stained with silver using standard method and were photoed to decide the suspect mutant exons of cases by comparing the discrepancy between band patterns of exons of cases and the corresponding exons of controls. The exons with positive discrepancy needed to be repeated once or twice as above.

\section{T-cloning and sequencing}

PCR products with suspect mutations and the corresponding ones of controls were simultaneously cloned directly into pGEM-T vector (Promega, USA). 10 random clones of each PCR product were selected. Bacterial culture liquids from these clones were used as templates to perform PCRSSCP followed by silver staining again in order to confirm the clones carrying the mutations. The plasmid DNAs were extracted from the clones carrying the mutations and the clones from corresponding controls and sequenced.

\section{Gelatin zymography}

A volume of $1 \mathrm{ml}$ of fresh blood from each patient was collected into a clean microcentrifuge tube, placed at room temperature for half an hour and centrifuged at 4500 r.p.m. for $15 \mathrm{~min}$ at $4^{\circ} \mathrm{C}$. The serum, straw yellow supernatant was adopted carefully for experiments. General density of the protein of the serum was quantified using BCA-200 Protein Assay kit (PIERCE, Rockford, USA). MMP-2 and MMP-9 enzymatic activity of the serum were assessed by gelatin zymography following the methods described previously. ${ }^{9}$ The activity of MMP-2 and MMP-9 were evaluated and relatively quantified using Lab Works Image Acquisition and Analysis Software (UVP Inc., UK) by setting the activity of one sample from normal controls to 1.0. Normalized activities were statistically analyzed by Independent-samples $t$-test in SPSS10.0 software (SPSS Inc., Chicago, IL, USA). Comparisons of means giving $P$-values with associated probabilities of difference of $<0.01$ were considered to be significantly different.

\section{Results}

We screened 32 cases with OA and 50 controls with only bone fracture using PCR-SSCP. Abnormally migrating bands were detected from the sample of a case (Figure 1a). The sequencing analyses revealed a nucleotide alteration of a

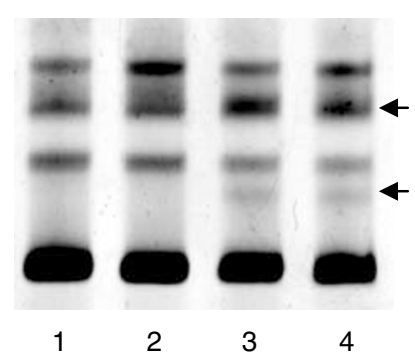

b

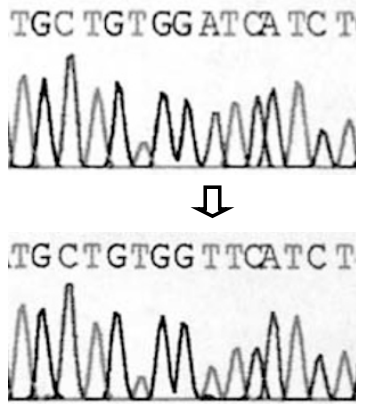

Figure 1 A mutation in the exon 4 of the Smad3 gene was identified in OA. (a) PCR-SSCP analyses of the Smad3 gene in patients with OA. Exon 4 was analyzed for mutation. Abnormal migrating bands (marked with arrowheads) were detected in one patient with OA (lanes 3 and 4). Lane 1 from control, and lane 2 from a case. (b) Detection of the Smad3 mutation by sequencing. Portions of sequencing data resulting from the analysis of PCR fragments of a control (upper) and the case with mutation (lower). The missense mutation $(A \rightarrow T)$ was marked by arrow.

the Smad3 gene in exon 4 (Figure 1b). The single base mutation was a non synonymous $\mathrm{A} \rightarrow \mathrm{T}$ difference in the position 2 of codon 197. This base change results in an asparagine to isoleucine amino-acid substitution. The mutation position is located in the linker region of the SMAD3 protein.

Recent studies have shown that higher expression of MMPs is a useful biochemical marker for joint destruction. ${ }^{10}$ In this study, we examined the proMMP-2 (72 kDa) and pro-MMP-9 $(92 \mathrm{kDa})$ activities in sera of patients with OA by gelatin zymography and found that the activities of pro-MMP2/9 were significantly higher than that of controls $(P<0.01)$ (Figure 2$)$. The activity of pro-MMP2/9 (normalized activities of MMP-2/9, 16.4/11.1) in serum of the patient with the Smad3 mutation was higher than the average activities of OA patients (mean value of normalized activities of MMP-2/9, $8.3 \pm 1.0 / 8.4 \pm 1.1, n=27$ ) and controls (mean value of normalized activities of MMP-2/9, 4.9 $\pm 0.4 / 4.1 \pm 0.4$, $n=30$ ) (Figure 2).

\section{Discussion}

Many studies have revealed that TGF- $\beta$ signals play important roles in maintaining normal status of articular cartilage, especially, TGF- $\beta 1$ have been implicated in human OA. ${ }^{11}$ Previous targeting experiments have shown that loss of $S m a d 3$ results in impaired immune responses, accelerated wound healing, decreased bone density, OA and access to colon cancer. ${ }^{12}$ Many attempts to screen Smad3 gene mutations in human cancers fail to find any missense mutation in human ovarian cancers, colorectal cancers or juvenile polyposis syndromes, indicating that 
a

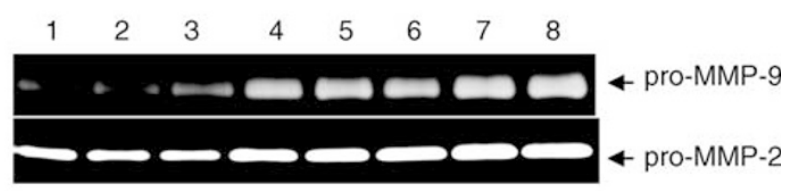

b

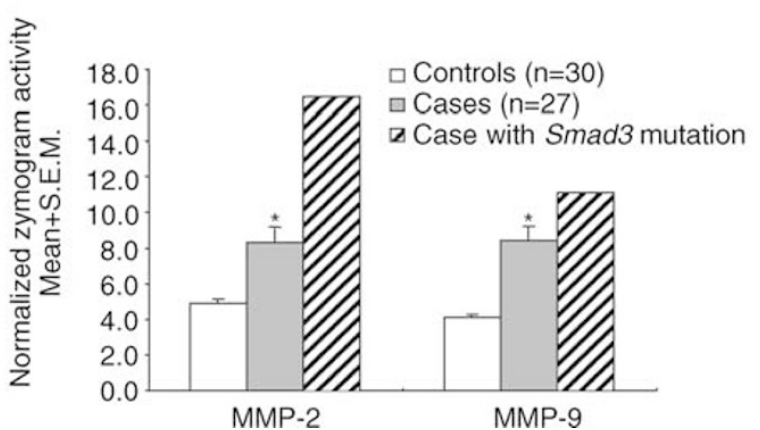

Figure 2 Higher expression of pro-MMP-2 and pro-MMP-9 was detected in serum of OA patient with Smad3 mutation. A, The activities of pro-MMP-2 (72 kDa) and pro-MMP-9 $(92 \mathrm{kDa})$ were detected by gelatin zymography. Same amounts of sera (2500 ng for MMP-2 and $40000 \mathrm{ng}$ for MMP-9) from representative samples were loaded in each well. Both brightness and area of bands of the patient with the mutation (lanes 7 and 8) are obviously higher than those other patients (lanes 4-6) and controls (lanes 1-3). B, The densitometric quantification of pro-MMP2/9 activities. The activity data from all serum samples analyzed by zymography were normalized by setting one sample in the control group to 1.0. The columns show the results (means+ standard error of the mean [SEM]) from controls $(n=30)$, cases $(n=27)$ and the case with Smad3 mutation. Asterisks indicated the statistically significant differences between controls and cases, $P<0.01$.

the mutations in the Smad3 gene are unlikely to be involved in human tumorigenesis. ${ }^{8,13-16}$ In this study, we found a single base mutation accompanied by amino-acid substitution in one patient with OA, suggesting that the Smad3 gene mutation is a possible predisposing factor for human OA. However, the low frequency $(1 / 32,3.13 \%)$ of Smad3 mutation also indicated that the TGF- $\beta / \operatorname{Smad} 3$ signaling might not be the major pathway involved in the onset of $\mathrm{OA}$ in human.

SMAD proteins consist of highly conserved amino- and carboxy-terminal domains separated by a more divergent linker region. Although the linker region is not well conserved, it is important for SMADs function and does have peptide motifs. ERK MAP-kinase phosphorylation sites were found within the linker region. ${ }^{17}$ Recent studies have shown that dexamethasone inhibits TGF- $\beta$-induced type II collagen mRNA expression through selective inhibition of ERK integrated AP-1 activation in cultured rat articular chondrocytes. ${ }^{18}$ The missense mutation $(\mathrm{P} 197 \mathrm{~N} \rightarrow \mathrm{I})$ we reported in this study was located in the linker region of SMAD3. Our previous studies have shown that Smad3 may mediate TGF- $\beta 1$ to inhibit the expression of MMP-9. ${ }^{19}$ In this study, we found that the patient carrying the mutation expressed a higher level of MMP-2 and MMP-9, suggesting that SMAD3 carrying this mutation may lead to upregulation of MMPs and eventually the development of OA. All these need to be further testified.

To our knowledge, this was the first report that Smad3 gene mutation was found in OA, providing insight into the function of SMAD3 mediated TGF- $\beta$ signals in the development of OA. Our results suggested that Smad3 gene mutation may be a risk factor for genetic susceptibilities to OA.

\section{Acknowledgements}

This work was supported by National Science Fund for Distinguished Young Scholars (30025028), National Natural Science Foundation of China (39970413) and Hi-Tech Research and Development Program of China (2001AA216081).

\section{References}

1 Jordan JM, Kington RS, Lane NE et al: Systemic risk factors for osteoarthritis; In Felson DT (conference chair): Osteoarthritis: new insights. Part 1: The disease and its risk factors. Ann Intern Med 2000; 133: 637-639.

2 Reginster JY: The prevalence and burden of arthritis. Rheumatology 2002; 41(suppl 1): 3-6.

3 Zeng Q, Huang S, Xiao Z et al: Osteoarthritis: clinical and epidemiological investigation. Chin J Internl Med 1995; 34: 88-90.

4 Zhang N, Shi Q, Zhang X et al: Epidemiological investigation of osteoarthritis. Chin J Intern Med 1995; 34: 84-87.

5 Massague J: TGF- $\beta$ signal transduction. Annu Rev Biochem 1998; 67: $753-791$.

6 Yang X, Letterio JJ, Chen L et al: Targeted disruption of SMAD3 results in impaired mucosal immunity and diminished $\mathrm{T}$ cell responsiveness to TGF- $\beta$. EMBO J 1999; 18: 1280-1291.

7 Yang X, Chen L, Xu L et al: TGF-beta/Smad3 signals repress chondrocyte hypertrophic differentiation and are required for maintaining articular cartilage. Journal of Cell Biology 2001; 153: $35-46$.

8 Arai T, Akiyama Y, Okabe S et al: Genomic structure of the human Smad3 gene and its infrequent alterations in colorectal cancers. Cancer Lett 1998; 122: 157-163.

9 Kleiner DE, Stetler-Stevenson WG: Quantitative zymography: detection of picogram quantities of gelatinase. Anal Biochem 1994; 218: 325-329.

10 Tanaka A, Kumagai S, Kawashiri S et al: Expression of matrix metalloproteinase-2 and -9 in synovial fluid of the temporomandibular joint accompanied by anterior disc displacement. J Oral Pathol Med 2001; 30: 59-64.

11 Yamada Y, Okuizumi H, Miyauchi A et al: Association of transforming growth factor betal genotype with spinal osteophytosis in Japanese women. Arthritis Rheum 2000; 43: 452-460.

12 Micheal W, Yang X, Deng C: Functions of mammalian Smad genes as revealed by targeted gene disruption in mice. Cytokine Growth Factor Rev 2000; 11: 49-58.

13 Bevan S, Woodford-Richens K, Rozen P et al: Screening SMAD1, SMAD2, SMAD3, and SMAD5 for germline mutations in juvenile polyposis syndrome. Gut 1999; 45: 406-408.

14 Wang D, Kanuma T, Takama F et al: Mutation analysis of the Smad3 gene in human ovarian cancers. Int J Oncol 1999; 15: 949-953.

15 Giggins GJ, Kinzler KW, Vogelstein B et al: Frequency of Smad gene mutations in human cancers. Cancer Res 1997; 57: 2578-2580. 
16 Osawa H, Shitara Y, Shoji $\mathrm{H}$ et al: Mutational analysis of transforming growth factor $\beta$ type II receptor, Smad2, Smad3 and Smad4 in esophageal squamous cell carcinoma. Int J Oncol 2000; 17: 723-728.

17 Kretzschmar M, Doody J, Massague J: Opposing BMP and EGF signaling pathways converge on the TGF- $\beta$ family mediator Smad1. Nature 1997; 389: 618-622.
18 Miyazaki Y, Tsukazaki T, Hirota Y et al: Dexamethasone inhibition of TGF beta-induced cell growth and type II collagen mRNA expression through ERK-integrated AP-1 activity in cultured rat articular chondrocytes. Osteoarthritis Cartilage 2000; 8: 378-385.

$19 \mathrm{Wu}$ Y, Feng Y, Yang X et al: TGF- $\beta 1$ mediated by SMAD3 inhibits the expression of MMP9 in COS7 cells. Chin J Biochem Mol Biol 2002; 18: 684-687. 